\title{
Study on the Influence of an Axial Distance Between Rotors on a Performance of a Small Counter-Rotating Wind Turbine
}

\author{
Michał Pacholczyk, Krzysztof Blecharz, Dariusz Karkosiński \\ Gdańsk University of Technology \\ Narutowicza 11/12, Gdańsk, Poland \\ michal.pacholczyk@pg.edu.pl; krzysztof.blecharz@pg.edu.pl; dariusz.karkosinski@pg.edu.pl
}

\begin{abstract}
In this paper, results of the investigation on the performance of a small counter-rotating wind turbine has been presented. Computational Fluid Dynamics methods have been used for wind turbine simulations. Rotors representation were introduced into computational domain by means of Actuator Line Model. Influence of an axial distance of CRWT rotors has been investigated. In all studied configurations rotors were counter-rotating with exactly the same rotational speed. Results of a study revealed noticeable increase in power coefficient for optimal configuration. Dynamic interaction between rotors has been investigated exposing significant interference in both torque and power in part of considered cases. Reasonable rotors distances, as well as rotors placements on tower's sides have been proposed. Used calculation method has been found to be appropriate for counter-rotating wind turbine simulation.
\end{abstract}

Keywords: Wind Energy, Counter-Rotating Wind Turbine, Computational Fluid Dynamics, Actuator Line Model.

\section{Introduction}

Counter-Rotating Wind Turbine (CRWT) is a two rotor turbine. Its second, counter-rotating rotor is placed in first rotor wake in order to extract part of an energy left in the wind. Although turbine's blades careful design, large portion of a wind energy is not being captured due to the physical limits. In case of Single Rotor Wind Turbine (SRWT) extraction efficiency is limited to $59.3 \%$, as classical Betz's theory states [1]. In real world operation turbine's efficiency reaches $40-50 \%$ [2]. As Newman showed in [3] and in [4] theoretical power extraction maximum for CRWT can be extended to $64 \%$ for 2 rotors and to $66.7 \%$ in case of an infinite number of rotors. Due to potential power production increase, CRWT concept is being widely investigated.

There few two CRWT configurations possible. Both rotors can be placed at upwind side, as for example in [5]. Such a configuration commonly implies small axial distance between rotors. When rotors are placed at upwind and downwind side axial distance can be much larger but it may results in massive hub construction. Such a configuration has been proposed and investigated for example in [6]. Combination of different rotor sizes is often proposed (see for example [7]). Smaller rotor can be located at the upwind or downwind large one's side. When considering electrical generator there are generally two approaches. First is to couple rotors shaft by differential mechanism attached to single conventional generator (e.g. [8], [9]). Second is to attached rotors shafts directly to separate, counter-rotating armatures. Such an approach, studied e.g. in [10] or [11], due to higher relative rotational speed can help to reduce generator size or volume of magnetic materials used. More comprehensive review of CRWT configuration and conducted investigations is provided in [12].

Prior to experimental investigation, numerical simulations with Computational Fluid Dynamics (CFD) are often performed. In general, if one wants to simulate wind turbine with CFD, he has to deal with 3-dimensional, unsteady, highly turbulent flows. There are several approaches to representation of a wind turbine's rotor in computational domain, accurately differentiated in [13]. Rotor's geometry can be recreated onto numerical grid, but extremely dense mesh in boundary layers is required. In such an approach, at the tip of the blade, compressibility can occur resulting in additional terms in solving equations. Therefore, several simplified methods have been proposed including Actuator Disk Model or Actuator Surface Model. In present study Actuator Line Model (ALM) introduced in [14] has been used since its allow to represent single blades at the relatively low computational cost.

Results of a CRWT parametric study has been presented in this paper. As parameter, axial distance between rotors has been chosen. Performance parameters have been compared with reference SRWT. Analysed CRWT' rotors has been exactly 
counter-rotating in all the cases. Similar study has been described in [15] but in present investigation more sophisticated turbulence modelling has been used. Turbine diameter is also much smaller.

\section{Methodology}

\subsection{Actuator Line Model}

Actuator Line Method methods represent rotor by a set of discrete segments distributed along each blade's span. At the centre of each segment body forces are applied on a fluid flow. This approach does not require modification of the computational grid but fully structurized one can be utilized. Based on the location and width $w$ of the segment, airfoil chord $c$ and twist, local wind speed $U_{r e l}$ and tabulated lift $C_{L}$ and drag $C_{D}$ coefficient values at the determined angle of attack $\alpha$, drag $\boldsymbol{D}$ and lift $\boldsymbol{L}$ can be calculated as follows:

$$
\begin{aligned}
& \boldsymbol{L}=\frac{1}{2} C_{L}(\alpha) \rho U_{r e l}^{2} c w, \\
& \boldsymbol{D}=\frac{1}{2} C_{D}(\alpha) \rho U_{r e l}^{2} c c
\end{aligned}
$$

Applied body force is equal and opposite in direction to vector sum of lift and drag. In order to project it in on surrounding finite elements Gaussian function is used:

$$
\boldsymbol{f}_{\text {proj }}=\frac{\boldsymbol{F}}{\varepsilon^{3} \pi^{3 / 2}} \exp \left[-(r / \varepsilon)^{2}\right]
$$

where $\boldsymbol{F}$ is calculated force, $\varepsilon$ Gaussian projection width and $r$ distance from the centre of an actuator segment.

Proper selection of ALM model parameters is crucial for accurate power production prediction. Most important is projection width $\varepsilon$. If too high value is selected force is projected far beyond actual blade swept area. From the other hand too low value results in discontinuous force distribution. General rule is that using $\varepsilon \leq 2 \Delta x$, where $\Delta x$ is finite element edge size, may introduce numerical instabilities into solver but often few trials are required to determine proper value. Other important factors are simulation time step and grid resolution as well as number of the actuator points along blade. Sufficient number of actuation points ensures continuous distribution of force while short time step is required to maintain a smooth application of a body force. Best practices and guidelines for simulation with ALM have been presenter e.g. in [16].

\subsection{Numerical Solver}

In this study Computational Fluid Dynamics methods have been used. Fluid motions, partial differential equations are solved by open source toolbox OpenFOAM (Open Fields Operation and Manipulation). ALM method has been implemented into OpenFOAM by Matt Churchfield and Sang Lee from NREL (a national laboratory of the U.S. Department of Energy, Office of Energy Efficiency and Renewable Energy). Created tool has been named SOWFA (Simulator fOr Wind Farm Applications) [17]. Calculations were carried out at the Academic Computer Centre in Gdańsk.

Large Eddy Scale (LES) turbulent flows simulation method have been chosen and used in CFD solver. This approach is based on the assumption that only large turbulent structures are numerically solved while those smaller than grid size are modelled with appropriate sub-grid scale models. Navier-Stokes equations are then converted to filtered form:

$$
\begin{gathered}
\nabla \cdot \widetilde{\boldsymbol{u}}=0 \\
\frac{\partial \widetilde{\boldsymbol{u}}}{\partial t}+(\widetilde{\boldsymbol{u}} \cdot \nabla) \widetilde{\boldsymbol{u}}=-\frac{1}{\rho} \nabla \tilde{p}+\nu \nabla^{2} \widetilde{\boldsymbol{u}}-\nabla \cdot \tau_{S G S}+\frac{1}{\rho} \overrightarrow{\boldsymbol{G}}
\end{gathered}
$$


where $\tau_{S G S}=-v_{S G S}\left(\nabla \widetilde{\boldsymbol{u}}+(\nabla \widetilde{\boldsymbol{u}})^{T}\right)$ and $v_{S G S}$ is the sub-grid scale viscosity. In this study standard Smagorinsky sub-grid model is used [18]. $\tilde{p}$ is filtered pressure, $\widetilde{\boldsymbol{u}}$ is filtered velocity vector and $v$ is kinematic viscosity. $\overrightarrow{\boldsymbol{G}}$ is external forces vector.

\subsection{Simulation Setup}

Computational domain is presented in the Figure 1. It is cube of dimensions 10D x 10D x 10D, where D is rotor diameter. Boundary conditions are set as shown in figure. Wind turbine representation is placed around 4D from uniform velocity inlet. In order to provide sufficient mesh density in turbine proximity, several levels of grid refinement is used. Finest mesh region has cylindrical shape with height of 4D and 2D diameter. Total element count is $9.573 \mathrm{mln}$ with finest element edge size of $0,0175 \mathrm{~m}$. Domain has been discretized in space and time with second order schemes. LES method with standard Smagorinsky model has been utilized and PISO algorithm has been used to sequentially solve turbulent flow equations. Input wind velocity was set to $8 \mathrm{~m} / \mathrm{s}$.

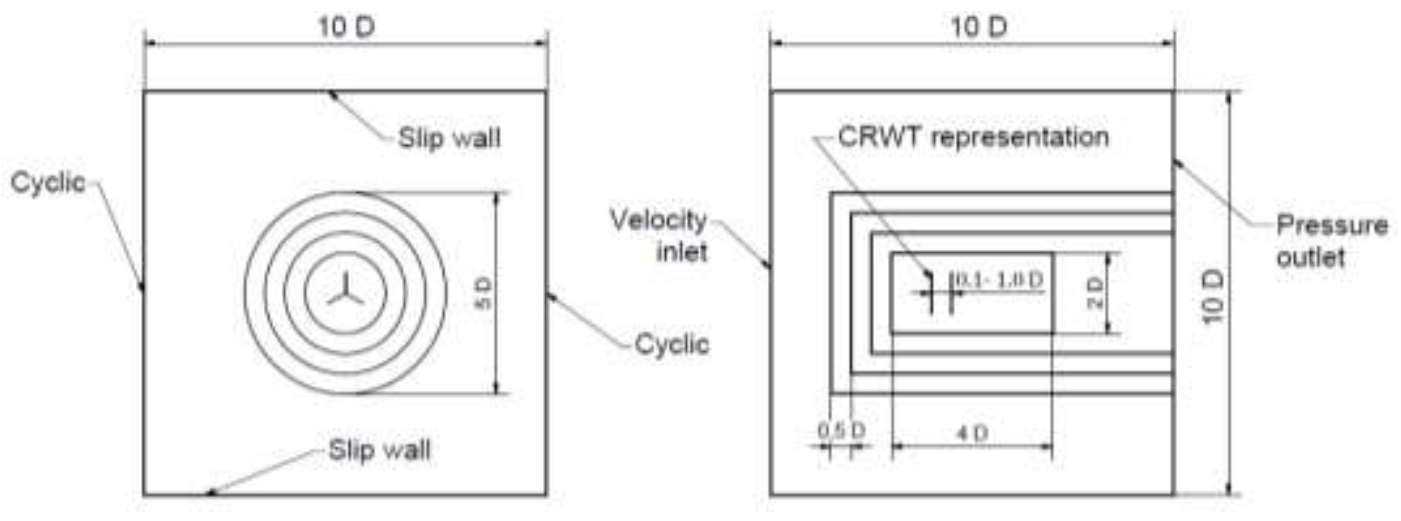

Fig. 1: Computational domain with boundary conditions.

Following ALM model's parameters have been used: Gaussian projection width $\varepsilon=0.035 \mathrm{~m}$; along blade span 40 finite elements are present and 80 actuator points were introduced; total simulation time was set to $5 \mathrm{~s}$ with time step $0.0002 \mathrm{~s}$. Results were averaged from $3 \mathrm{~s}$ to $5 \mathrm{~s}$. During simulation with TSR $=5$ each rotor perform over 45 revolution.

\section{Results}

\subsection{Reference Turbine Simulation}

In studied CRWT configurations both rotors are the same, newly designed 3-bladed rotors with tip diameter D $=1.4 \mathrm{~m}$ and root diameter equal to $0.25 \mathrm{~m}$. Airfoil NACA4418 has been selected and distributed optimally along blade's span for operation with TSR $=5$. Blade Element Momentum Theory (BEMT) has been used in design process. In order to verify numerical setup SRWT simulation with ALM method has been conducted. Results have been then confronted with BEMT calculations which is considered credible and representative approach to wind turbine's performance prediction. In Figure 2 power coefficient $C_{p}$ and thrust coefficient $C_{t}$ characteristics obtained with BEMT and ALM are compered. Results for $C_{t}$ coefficient are in very good agreement for studied TSR range. $C_{p}$ coefficient results from CFD calculation are slightly overestimated beyond TSR $=5$. Operational optimum is found for TSR $=6$ even though blade was designed and optimised for TSR $=5$. For designed TSR $=5, C_{p}$ coefficient obtained with ALM equals to 0.4767 and for BEMT 0.4729. Differences between both methods are small enough to be accepted. In the Figure 3 mean velocity and vorticity contours for operation with TSR $=5$ are shown. It can be noticed that wake is stable several diameters behind a rotor. With ALM method used, vorticities induced by single blade's tip are clearly visible. 


\subsection{CRWT Parametric Study Results}

With numerical setup validated parametrical study of CRWT has been carried out. In this investigation influence of the axial distance between rotors has been studied. In all cases rotors were exactly counter-rotating with TSR $=5$. Axial distance varied from $0.1 \mathrm{D}$ to $1 \mathrm{D}$. In total, 5 configurations have been considered $(0.1 \mathrm{D}, 0.25 \mathrm{D}, 0.5 \mathrm{D}, 0.75 \mathrm{D}$ and $1 \mathrm{D})$.
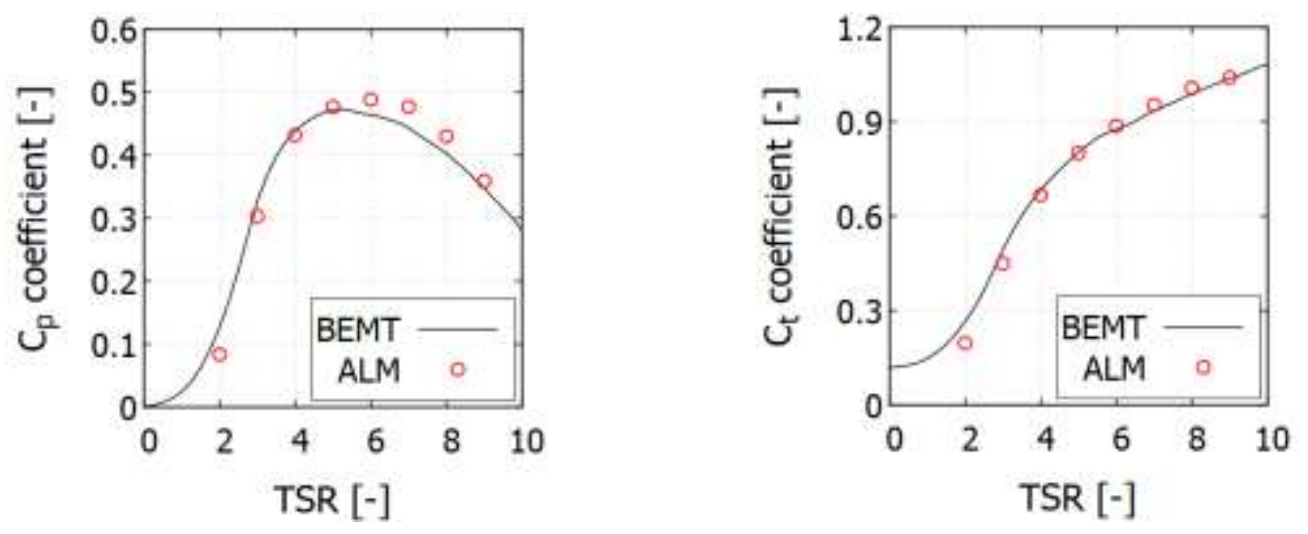

Fig. 2: Comparison of $\mathrm{C}_{\mathrm{p}}$ and $\mathrm{C}_{\mathrm{t}}$ coefficient calculation results obtained with BEMT and ALM.

Except of the case when axial distance was set to 0.1D in all studied CRWT configuration $\mathrm{C}_{\mathrm{p}}$ coefficient was higher than in reference SRWT. Its value varied from 0.4740 for $0.1 \mathrm{D}$ case (decrease of $0.57 \%$ ) to 0.5149 for $1 \mathrm{D}$ case (increase of 8.01\%). Summary of $\mathrm{C}_{\mathrm{p}}$ coefficient calculation results have been presented in Figure 4. It is seen that power gain increase with axial distance. When analysing separate rotors performance it can be seen that front rotor produces most of the power in all CRWT configuration. In optimum 1D case front rotor $\mathrm{C}_{\mathrm{p}}$ is 0.4321 and rear is 0.0828 . Second rotor reduced first rotor's power of $9.36 \%$ in comparison to SRWT but in general power production gain is noticeable. Additional advantage is that due to rotors counter-rotation with the same tip speed ratio in all cases shaft relative rotational speed has been doubled. As expected, CRWT thrust is higher than in SRWT for all the cases. $C_{t}$ coefficient is lowest for case $1 \mathrm{D}\left(\mathrm{C}_{t}=1.1107\right.$, increase of $38.84 \%)$ and highest in case of $0.25 \mathrm{D}\left(\mathrm{C}_{\mathrm{t}}=1.1156\right.$, increase of $\left.39.45 \%\right)$.
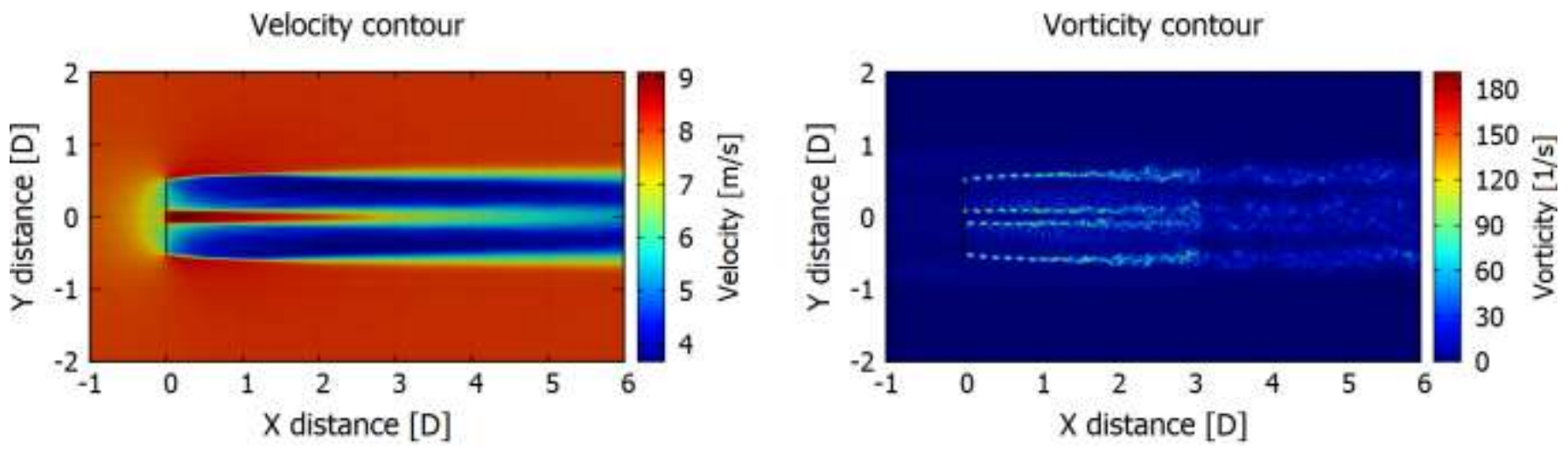

Fig. 3: SRWT mean velocity and vorticity contours for operation with TSR $=5$.

Unsteady fluid flow equations have been solved therefore it is possible to analyse dynamic performance of the CRWT. Thanks to representation of single blades in ALM approach, interactions between front and rear rotors can be observed. Torque and power plots in time domain for all studied configurations have been presented in Figure 5. Quarter of the last simulation second has been plotted. In that period each rotor performed slightly more than 2 revolutions. One can notice 
large fluctuation in both torque and power in cases $0.1 \mathrm{D}$ and $0.25 \mathrm{D}$. All the peaks correspond to moments when blades from front and rear rotors pass over each other. In case $0.1 \mathrm{D}$ torque fluctuations reach about $20-25 \%$ of average value. When axial distance is equal and higher than $0.5 \mathrm{D}$ dynamic interactions seem to disappear.

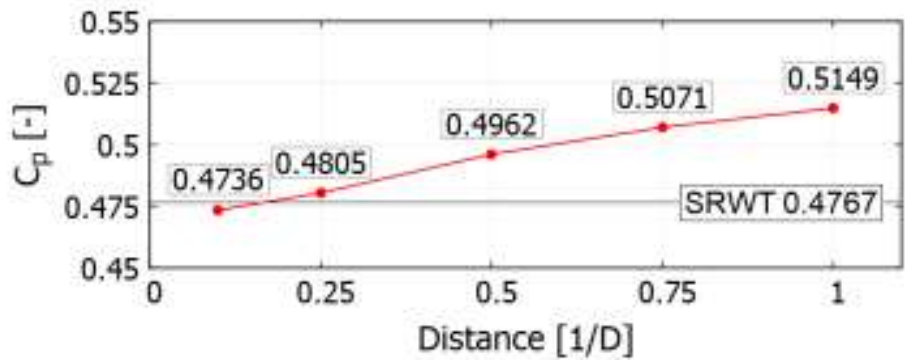

Fig. 4: Summary of $\mathrm{C}_{\mathrm{p}}$ coefficient values for all the studied configurations.

In the Figure 6 mean wind speed and instantaneous vorticity contours for all configurations have been showed. Wake behind second rotor for all scenarios seems to be similar and around 2-4D completely loosing stability. For all axial distances, as expected from consistent and high value of $\mathrm{C}_{t}$ coefficient, wake expands much more than in case of SRWT. Large reduction in wind speed is observed behind turbine. However, more detailed study is required in order to accurately investigate wake behind CRWT turbine. Denser mesh should be used for a wake region, domain should be extended as well.

Torque [Nm]

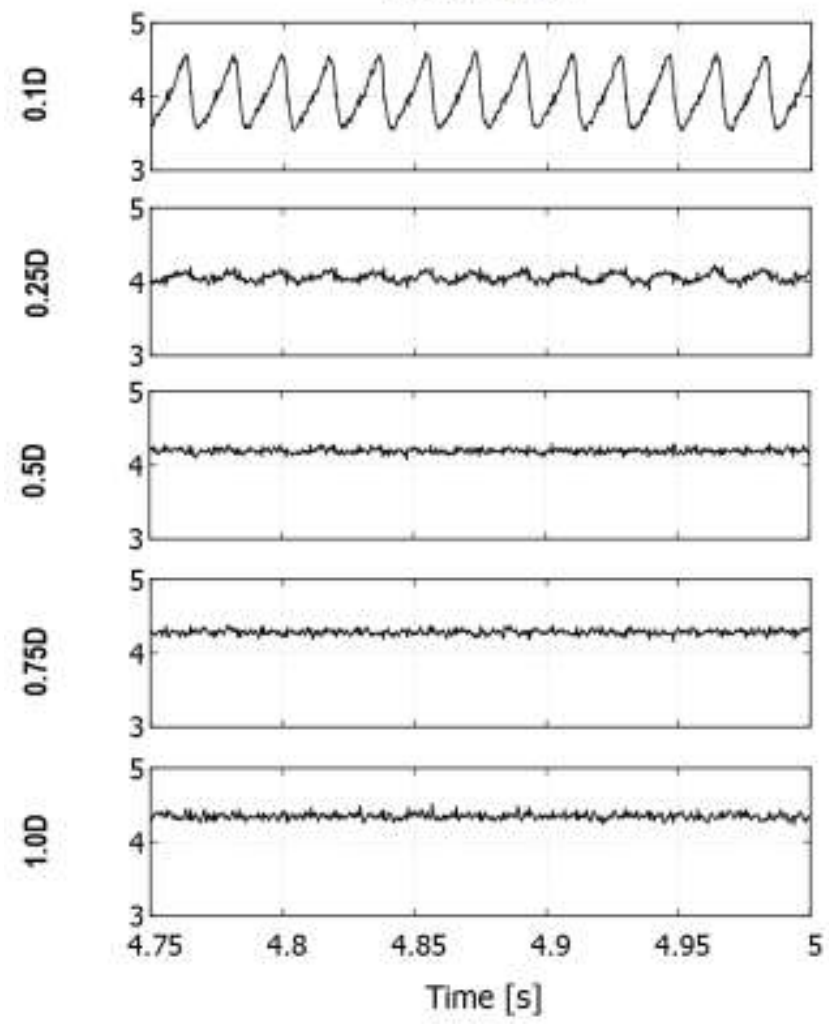

Power [W]

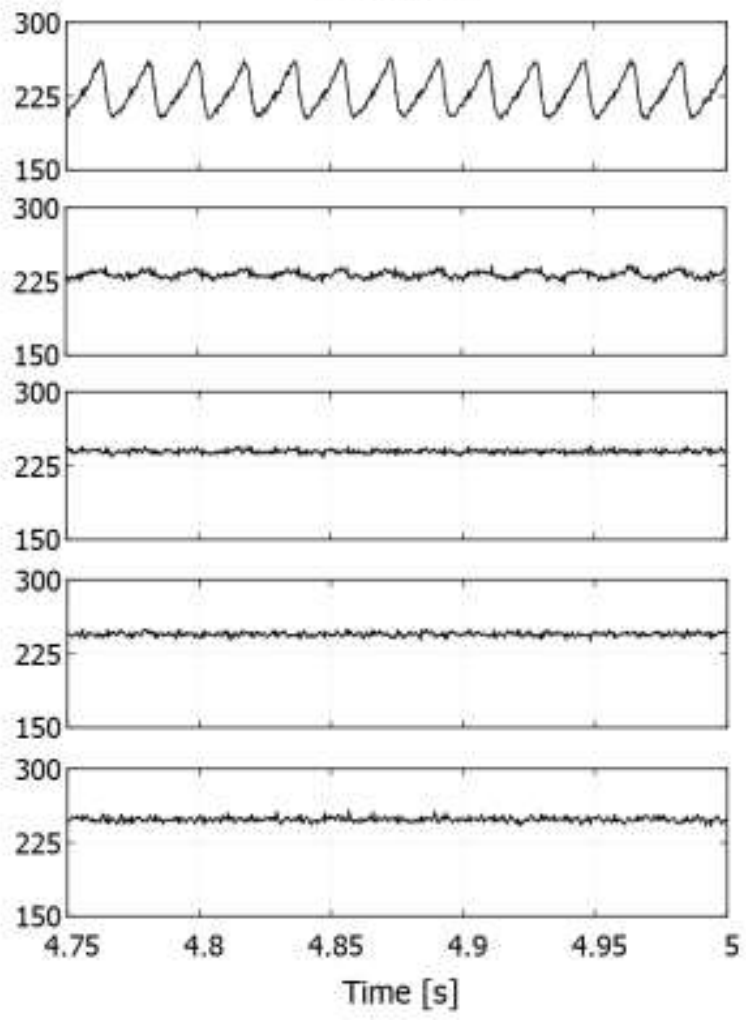

Fig. 5: Torque and power in time domain for all the CRWT configurations. 

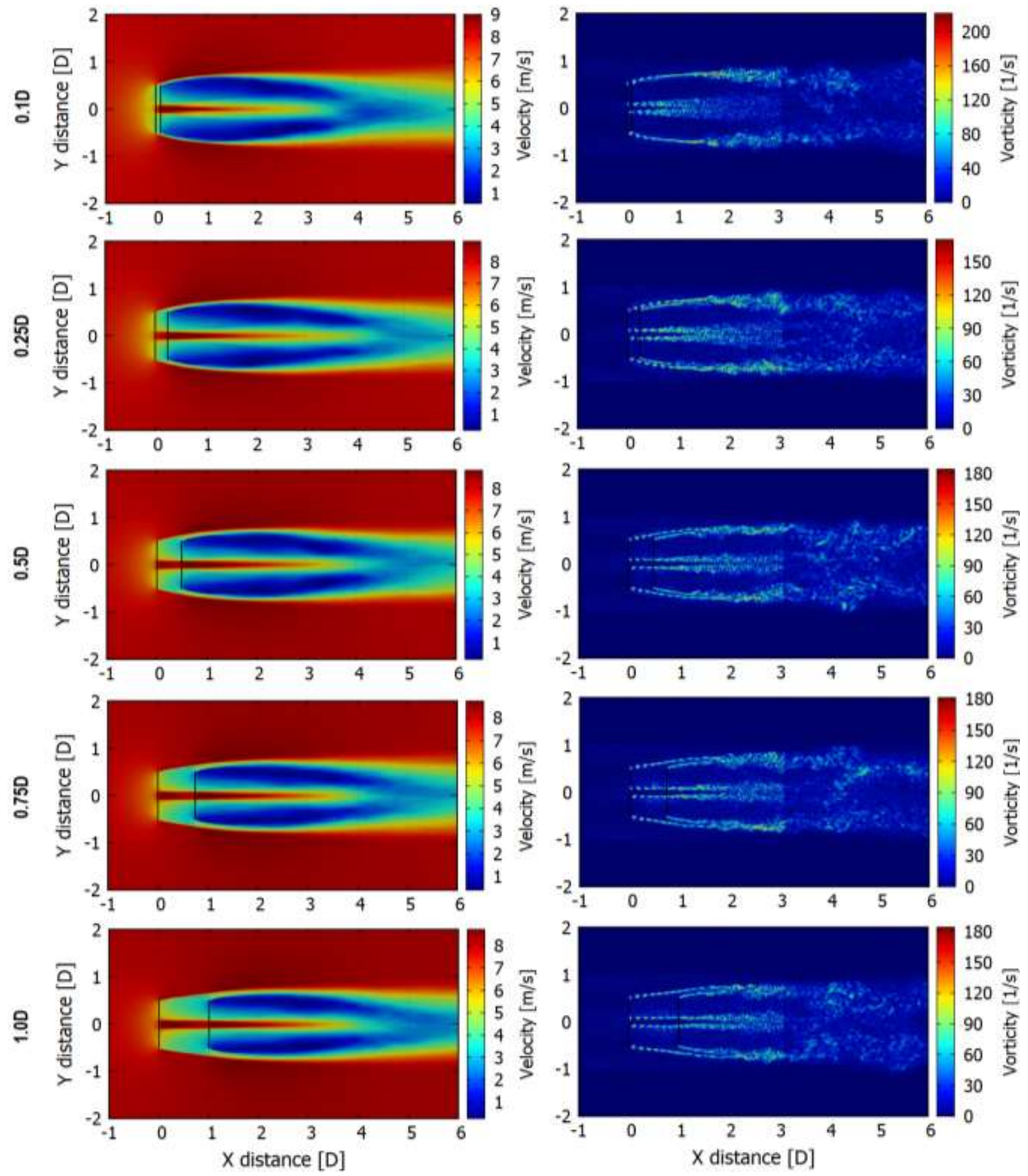

Fig. 6: Mean velocity and vorticity contours for all CRWT configurations. 


\section{Conclusion}

Parametrical CFD study on a small counter-rotating wind turbine performance has been carried out in this paper. Influence of an axial distance has been chosen as a studied parameter. In all configurations rotors counter-rotated with constant and equal rotational speed. Investigated axial distance range stretches from 0.1D to 1D. CRWT $\mathrm{C}_{\mathrm{p}}$ coefficient was was higher for all studied axial distances, except of a case when rotors were separated by 0.1D. Power gain extended from from $0.69 \%$ to $8.01 \%$, in comparison to reference SRWT. Optimal case was 1D. Power gain showed tendency to grow together with axial distance. Thrust coefficient, as expected, was much higher than in SRWT with value ranging from 1.1107 to 1.1156. Wake behind rear rotor was similar in all the cases. It expanded much more than in single rotor case and lost its stability much faster. When considering dynamic interaction between rotors, large fluctuations in torque and power can be observed for distance 0.1D. In case of torque, magnitude of fluctuation reaches 20-25\% of average value. Both with increase of an axial distance, fluctuation tends to decrease and disappearing from $0.5 \mathrm{D}$. Observed variations in dynamic performance were induced by rotors' blades passing each other.

Following conclusions can be established based on a conducted study: CRWT configurations with rotors separated by distance between $0.1 \mathrm{D}$ to $0.5 \mathrm{D}$ does not provide enough power gain to justify second rotor addition costs. Moreover, large dynamic interactions in torque and power plots in cases $0.1 \mathrm{D}$ and $0.25 \mathrm{D}$ would cause extreme fatigue loads on a turbine's construction as well as uneven power generation. As obtained results showed, CRWT' rotors distance should be contained in range from $0.5 \mathrm{D}$ to $1 \mathrm{D}$. Noticeable power gain is observed with maximum $8.01 \%$ for $1 \mathrm{D}$ case. No significant dynamic interactions are noticed for all the cases from that range. It has been shown that CRWT can be considered as affordable renewable energy source, with double shaft relative rotational speed.

Therefore, it can be concluded that CRWT configurations with rotors at the same side of a tower are not favourable since rotors distance equal and higher than $0.5 \mathrm{D}$ is not appropriate for this types of construction. Rational CRWT construction is that with rotors at upwind and downwind side. However, rotors distance more than 1D would lead to oversized hub design, even though power gain showed tendency to grow further beyond that distance. In the end, when considering CRWT prototyping it is reasonable to design one with rotors at different sides of a tower with rotors distance from range 0.5D-1D.

Actuator Line Model approach to CRWT simulations used in this study turn out to be computationally cost effective and able to reveal dynamic interactions between rotors. Future study should include investigation of different rotors rotational speeds for all axial distances, detailed investigation of near and far CRWT wake as well as tower influence investigation. Experimental verification of obtained results and ALM approach applied to CRWT should be performed.

\section{References}

[1] A. Betz, "Introduction to the Theory of Flow Machines," Introd. to Theory Flow Mach., 1966.

[2] P. J. Schubel and R. J. Crossley, "Wind turbine blade design," Energies, vol. 5, no. 9, pp. 3425-3449, 2012.

[3] B. G. Newman, "Actuator-disc theory for vertical-axis wind turbines," J. Wind Eng. Ind. Aerodyn., vol. 15, no. 1-3, pp. 347-355, Dec. 1983.

[4] B. G. Newman, "Multiple actuator-disc theory for wind turbines," J. Wind Eng. Ind. Aerodyn., vol. 24, no. 3, pp. 215-225, 1986.

[5] L. Romański, J. Bieniek, P. Komarnicki, M. Dębowski, and J. Detyna, "Operational Tests of a Dual-Rotor Mini Wind Turbine,” Eksploat. i Niezawodn. - Maint. Reliab., vol. 18, no. 2, pp. 201-209, 2016.

[6] R. W. Y. Habash, V. Groza, Y. Yang, C. Blouin, and P. Guillemette, "Performance Testing and Control of a Small Wind Energy Converter,” 2011 Sixth IEEE Int. Symp. Electron. Des. Test Appl., pp. 263-268, 2011.

[7] T. Kanemoto and A. M. Galal, "Development of Intelligent Wind Turbine Generator with Tandem Wind Rotors and Double Rotational Armatures (1st Report, Superior Operation of Tandem Wind Rotors )," JSME Int. J., vol. 49, no. 2, pp. 450-457, 2006.

[8] R. Herzog and L. Mûriers, "Performance and Stability of a Counter-Rotating Windmill Using a Planetary Gearing : Measurements and Simulation," no. April, p. 12003, 2010.

[9] R. W. Y. Habash, V. Groza, Y. Yang, C. Blouin, and P. Guillemette, "Performance of a Contrarotating Small Wind Energy Converter," ISRN Mech. Eng., vol. 2011, pp. 1-10, 2011. 
[10] L. A. Mitulet, G. Oprina, R. A. Chihaia, S. Nicolaie, A. Nedelcu, and M. Popescu, "Wind tunnel testing for a new experimental model of counter-rotating wind turbine," Procedia Eng., vol. 100, no. January, pp. 1141-1149, 2015.

[11] P. Sutikno and D. B. Saepudin, "Design and Blade Optimization of Intelligent Wind Turbine," Most, no. December, pp. 1-15, 2010.

[12] G. Oprina, R. A. Chihaia, S. Nicolaie, C. A. Băbuțanu, and A. Voina, "A Review on Counter-Rotating Wind Turbines Development," J. Sustain. Energy, vol. 7, no. 3, pp. 91-98, 2016.

[13] B. Sanderse, S. P. Pijl, and B. Koren, "Review of computational fluid dynamics for wind turbine wake aerodynamics," Wind Energy, vol. 14, no. 7, pp. 799-819, Oct. 2011.

[14] J. N. Sørensen and W. Z. Shen, "Numerical Modelling of Wind Turbine Wakes," J. Fluids Eng., vol. 124, no. 2, pp. 393-399, 2002.

[15] W. Z. Shen, V. A. K. Zakkam, J. N. Sørensen, and K. Appa, “Analysis of counter-rotating wind turbines," J. Phys. Conf. Ser., vol. 75, no. 1, 2007.

[16] L. Martinez, S. Leonardi, M. Churchfield, and P. Moriarty, "A Comparison of Actuator Disk and Actuator Line Wind Turbine Models and Best Practices for Their Use," 50th AIAA Aerosp. Sci. Meet. Incl. New Horizons Forum Aerosp. Expo., no. October 2016, 2012.

[17] "NWTC Information Portal (SOWFA)." [Online]. Available: https://nwtc.nrel.gov/SOWFA. [Accessed: 25-Aug2018].

[18] J. Smagorinsky, "General Circulation Experiments with the Primitive Equations," Mon. Weather Rev., vol. 91, no. 3, pp. 99-164, Mar. 1963. 